\title{
Sensitivity of Polycrystal Plasticity to Slip System Kinematic Hardening Laws for Al 7075-T6
}

\author{
Conor Hennessey ${ }^{1}$, Gustavo M. Castelluccio², and David L. McDowell ${ }^{1,3}$
}

1 Woodruff School of Mechanical Engineering, Georgia Institute of Technology, Atlanta, GA, USA

2 Sandia National Laboratories, Albuquerque, NM, USA

${ }^{3}$ School of Materials Science and Engineering, Georgia Institute of Technology, Atlanta, GA, USA

\section{Abstract}

The prediction of formation and early growth of microstructurally small fatigue cracks requires use of constitutive models that accurately estimate local states of stress, strain and cyclic plastic strain. However, little research has attempted to systematically consider the sensitivity of overall cyclic stressstrain hysteresis and higher order mean stress relaxation and plastic strain ratcheting responses introduced by the slip system back-stress formulation in crystal plasticity, even for face centered cubic (FCC) crystal systems. This paper explores the performance of two slip system level kinematic hardening models using a finite element crystal plasticity implementation [1]. The two kinematic hardening formulations aim to reproduce the cyclic deformation of polycrystalline Al 7075-T6 in terms of both macroscopic cyclic stress-strain hysteresis loop shape, as well as ratcheting and mean stress relaxation under strain- or stress-controlled loading with mean strain or stress, respectively. The first formulation is an Armstrong-Frederick type hardening-dynamic recovery law for evolution of the back stress [2]. This approach is capable of reproducing observed deformation under completely reversed uniaxial loading conditions, but overpredicts the rate of cyclic ratcheting and associated mean stress relaxation. The second formulation corresponds to a multiple back stress Ohno-Wang type hardening law [3] with nonlinear dynamic recovery. The adoption of this back stress evolution law greatly improves the capability to model experimental results for polycrystalline specimens subjected to cycling with mean stress or strain. The relation of such nonlinear dynamic recovery effects are related to slip system interactions with dislocation substructures.

\section{Introduction}

The over-prediction of cyclic strain accumulation or ratcheting under mean stress loading by the Armstrong-Frederick (AF) rule under asymmetric loading has been widely noted in the literature regarding $\mathrm{J}_{2}$ plasticity [3-7]. Thus, a similar concern is expected for an Armstrong-Frederick hardening law framed at the slip system level in crystal plasticity. However, few studies have investigated the model-form error of slip system level kinematic hardening laws in polycrystal plasticity due to the difficulties in identifying the relative roles of intergranular and intragranular contributions to back stress. Indeed, grain orientation and misorientation distributions can induce significant back stress contributions to polycrystalline stress-strain response in crystals with strong elastic anisotropy and/or low symmetry slip systems, for example HCP crystals. But Al alloys have mild elastic anisotropy and high symmetry FCC lattices that do not exhibit such pronounced intergranular interactions. Further, precipitate strengthened Al alloys are expected to manifest significant slip system level back stress by 
virtue of the internal stresses developed via dislocation-precipitate interactions. Therefore, the form of the kinematic hardening law at the slip system level is critical in modeling the constitutive response of this alloy system. In an effort to improve the ability to address not only first order stress-strain hysteresis behavior but also higher order phenomena such as ratcheting under applied mean stress and mean stress relaxation during cycling with a mean strain, another model is considered for the back stress evolution. This model corresponds to an extension of the work of Ohno and Wang for $\mathrm{J}_{2}$ plasticity [3] in accordance with the macroscopic model proposed by McDowell [4]. It is argued that consideration of ratcheting and mean stress relaxation is much more demanding in terms of slip system level model form, as necessary to address local states to evaluate crack formation in fatigue, for example.

Prior work using the crystal plasticity finite element method (CPFEM) for modeling response of polycrystalline Al 7075-T6 has considered isotropic hardening arising from dislocation/precipitate interactions, such as Orowan looping and forest dislocation strengthening [8-11]. Although such an approach can be fit to limited experimental data in terms of cyclic peak stresses, obtaining agreement in terms of the shape of the cyclic stress-strain curve is difficult without the adoption of kinematic hardening. When the slip system relation does not explicitly include kinematic hardening, the apparent kinematic hardening of simulated polycrystalline response emerges from intergranular interactions and is relatively weak for Al alloys. Wang et al. [12] and Johnston et al. [13] employed such an approach with combined isotropic and Armstrong-Frederick kinematic hardening. However, polycrystalline simulations we have conducted using the forms and parameter values published by Johnston et al. [13] were unable to match experimental cyclic stress-strain data due to the dominance of isotropic hardening over a slowly evolving kinematic hardening response. Review of previous CPFEM modeling work applied to Al 7075-T6 reinforces the need for significant levels of kinematic hardening at the slip system level to properly account for cyclic strain accumulation and cyclic plasticity at both the microscale and macroscale.

The crystal plasticity kinematics and slip system flow rule are first summarized, followed by the slip system kinematic hardening laws, computational setup, and model calibration schemes. The performance of the two models is compared, focusing on the ability of the Ohno-Wang model to match experimental stress-strain data under a wide range of loading conditions, including completely reversed stress-strain hysteresis loops as well as ratcheting and mean stress relaxation behaviors. Finally, a discussion of the results and implications from this work are presented, including an interpretation of origin of the nonlinear dynamic recovery characteristics of the Ohno-Wang model in terms of dislocation substructure evolution.

\section{Constitutive Framework}

The kinematic framework of the crystal plasticity formulation [14] considers the usual decomposition of the deformation gradient into elastic $\left(F^{e}\right)$, and plastic ( $\left.F^{p}\right)$ parts according to $F=F^{e} \cdot F^{p}$. The material time derivative of the plastic deformation gradient relates to the isoclinic intermediate configuration plastic velocity gradient $\hat{L}^{p}$ via,

$$
\dot{F}^{p}=\hat{L}^{p} \cdot F^{p}=\left(\sum_{\alpha=1}^{N_{\alpha}} \dot{\gamma}^{\alpha} s_{0}^{\alpha} \otimes m_{0}^{\alpha}\right) \cdot F^{p} .
$$


The plastic velocity gradient in the current configuration relates to the plastic velocity gradient of the intermediate configuration through the expression,

$$
L^{p}=F^{e} \cdot \sum_{\alpha=1}^{N_{\alpha}} \dot{\gamma}^{\alpha} s_{0}^{\alpha} \otimes m_{0}^{\alpha} \cdot\left(F^{e}\right)^{-1} .
$$

In Eqns. (1)-(2), $\dot{\gamma}^{\alpha}$ is the shearing rate on slip system $\alpha, s_{0}^{\alpha}$ and $m_{0}^{\alpha}$ are respectively unit vectors in the slip direction and slip plane normal direction in the isoclinic intermediate configuration; these are unchanged from the reference configuration. The $2^{\text {nd }}$ Piola-Kirchhoff stress, $T$, is obtained via the linearized elastic relation in the intermediate configuration given by,

$$
T=\bar{C}: E^{e},
$$

where $\bar{C}$ is the $4^{\text {th }}$ rank elasticity tensor and the elastic Green strain tensor is defined by,

$$
E^{e}=\frac{1}{2}\left[\left(\mathrm{~F}^{e}\right)^{T} \cdot \mathrm{F}^{e}-I\right] \text {. }
$$

Small elastic strain is typical, consistent with the linear relation in Eqn. (3). The Cauchy stress ( $\sigma$ ) is related to the $2^{\text {nd }}$ Piola-Kirchhoff stress tensor via,

$$
\sigma=\frac{1}{\operatorname{det}\left(\mathrm{F}^{e}\right)} \mathrm{F}^{e} \cdot T \cdot\left(\mathrm{F}^{e}\right)^{T}
$$

The resolved shear stress on slip system $\alpha, \tau^{\alpha}$, is given by,

$$
\tau^{\alpha}=\sigma:\left(s^{\alpha} \otimes m^{\alpha}\right) .
$$

The shearing rate on slip system $\alpha$ is related to the resolved shear stress through the flow rule,

$$
\dot{\gamma}^{\alpha}=\dot{\gamma}_{o}\left|\frac{\tau^{\alpha}-\chi^{\alpha}}{g^{\alpha}}\right|^{M} \operatorname{sgn}\left(\tau^{\alpha}-\chi^{\alpha}\right),
$$

where $\dot{\gamma}^{\alpha}$ is the shearing rate for slip system $\alpha, \dot{\gamma}_{o}$ is the reference shearing rate, and $\chi^{\alpha}$ and $g^{\alpha}$ are the corresponding slip system back stress and drag stress, respectively; $M$ is the inverse strain rate sensitivity exponent.

The kinematic hardening rate, i.e., evolution of the back stress, depends on the shearing rate on the current slip system and the current back stress. Additionally, both models assume that the drag stress on each slip system is held constant throughout the simulation, i.e., $g^{\alpha}=G_{o}$; isotropic hardening is neglected, representative of cyclically stable stress-strain response (cyclic softening or hardening transients due to the development of dislocation structures are neglected). The back stress evolution is assumed to be of purely self-hardening type.

The first model assigns the slip system back stress evolution to follow an Armstrong-Frederick [2] hardening-dynamic recovery form, i.e., 


$$
\dot{\chi}^{\alpha}=h \dot{\gamma}^{\alpha}-r \chi^{\alpha}\left|\dot{\gamma}^{\alpha}\right|
$$

where $h$ and $r$ are constants. The asymptotic saturation level of the slip system back stress corresponds to the condition $\dot{\chi}^{\alpha}=0$ and is given by,

$$
b=\frac{h}{r} \text {. }
$$

The second model incorporates a multiple back stress Ohno-Wang [3] type evolution law, adapted in essence to the crystal plasticity setting from a macroscopic model introduced by McDowell [4]. The evolution of slip system back stress in this model form is given by,

$$
\dot{\chi}_{i}^{\alpha}=h_{i} \dot{\gamma}^{\alpha}-r_{i}\left(\frac{\left|\chi_{i}^{\alpha}\right|}{b_{i}}\right)^{m_{i}} \quad \chi_{i}^{\alpha}\left|\dot{\gamma}^{\alpha}\right| \quad, \quad \dot{\chi}^{\alpha}=\sum_{i=1}^{2} \dot{\chi}_{i}^{\alpha} .
$$

Here, the total back stress is decomposed into two components $(i=1,2)$. Exponent $m_{i}$ controls the highly nonlinear character of the dynamic recovery (second) term, reducing its influence when the back stress is far from saturation levels. For high values of $m_{i}$, the OW back stress model evolves in nearly a piecewise linear manner. If $m_{i}=0$, the Ohno-Wang back stress evolution law reduces to the form of the Armstrong-Frederick equation. This work assumes the same value of $m_{1}=m_{2}$ for both terms of the back stress evolution; in general, different values could be used, but our objective is to assess model-forms rather than parameter optimization. The two kinematic hardening forms considered in this paper will be referred to as "AF" for the Armstrong-Frederick hardening law (Eqn. (8)) and "OW" for Ohno-Wang type back stress evolution model (Eqn. (10)).

\section{Model Calibration}

The elastic stiffness tensor, $C_{i j k l}$, is identical for both models. Here, we adopt the values for Al 7075-T6 from Bozek et al. [9], which provide good agreement with experimental results within the elastic regime. All material constants pertain to room temperature behavior. The values of the cubic crystal elastic constants are given by $\mathrm{C}_{11}=107.3 \mathrm{GPa}, \mathrm{C}_{12}=60.9 \mathrm{GPa}$, and $\mathrm{C}_{44}=28.3 \mathrm{GPa}$. For all models, $\dot{\gamma}_{o}=0.001 \mathrm{~s}^{-1}$.

The inverse strain rate sensitivity exponent in the flow rule was assigned as $M=150$ for model $\mathrm{AF}$ and $M=75$ for model $\mathrm{OW}$. A lower value of $M$ was employed for the $\mathrm{OW}$ model than for the $\mathrm{AF}$ model in order to improve convergence, and had a negligible effect on the constitutive response under quasi-static loading within the strain ranges of interest at room temperature. For comparison, Bozek et al. [7] employed an inverse strain rate sensitivity exponent of 200 .

The initial fitting of both models was conducted by comparing to completely reversed, cyclic stress-strain data obtained by Arcari [15] at strain amplitudes of $1 \%$ and $1.8 \%$; we assume that these responses are close to a cyclically stable condition. The simulations performed to match these results were conducted using a single cubic, periodic microstructure instantiation with approximately 700 
equiaxed grains with a $14 \mu \mathrm{m}$ mean grain diameter and random orientation distribution, as shown in Figure 1. This model is regarded as large enough to constitute a representative volume element (RVE) for purposes of reproducing cyclic stress-strain response, but is not necessarily large enough to serve as a RVE for assessment of fatigue crack formation based on local cyclic plastic strain states within individual grains. The side length of the cubic RVE was $100 \mu \mathrm{m}$ and the element size was $5 \mu \mathrm{m}$ (for a total of 8000 elements). The microstructure instantiation was subjected to uniaxial, quasi-static, straincontrolled cyclic loading with 3D periodic boundary conditions.

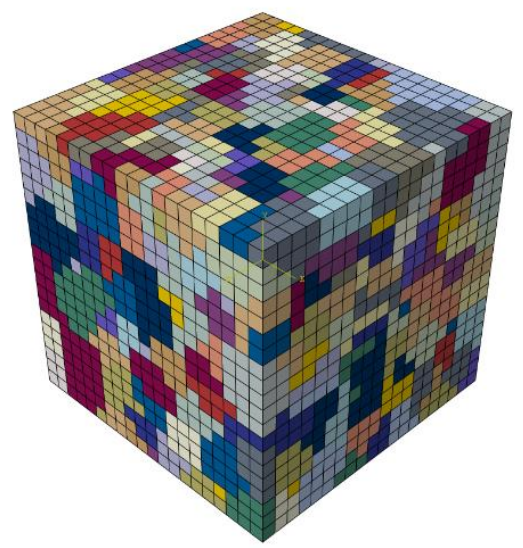

Figure 1. Summary of the mesh used in the simulations, consisting of approximately $\mathbf{7 0 0}$ grains with a $14 \mu \mathrm{m}$ mean grain diameter. Element size is $5 \mu \mathrm{m}$ and side lengths of the cube are $100 \mu \mathrm{m}$.
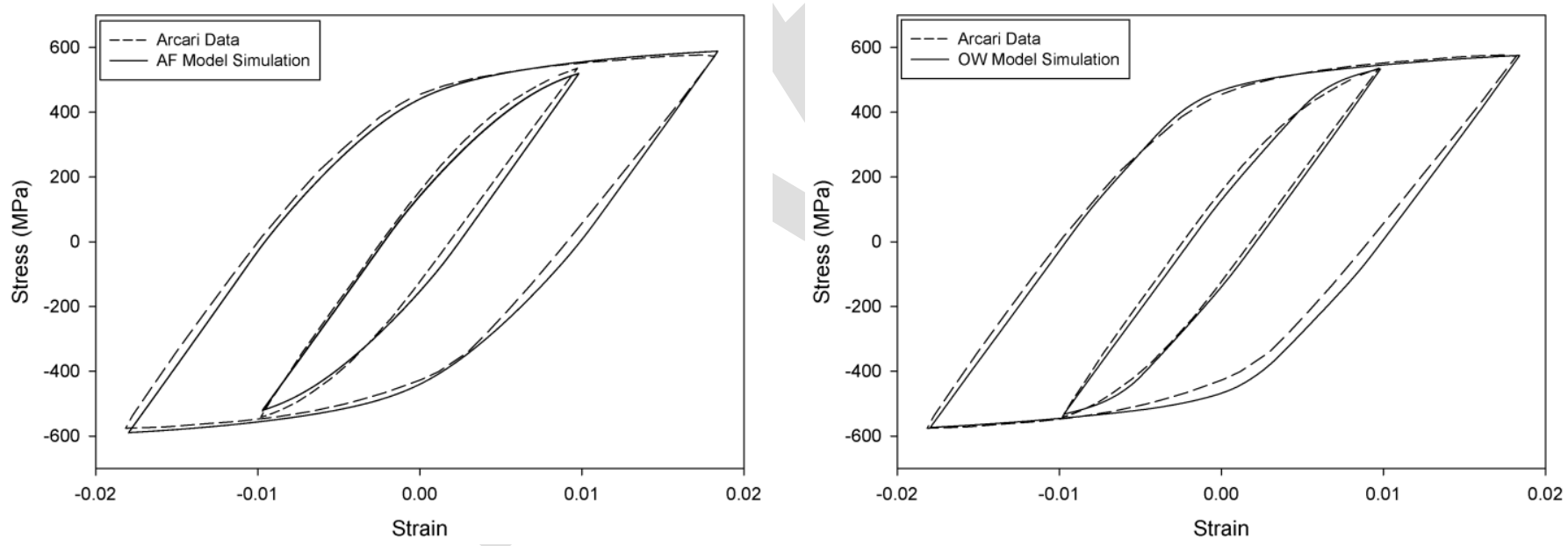

Figure 2. Left: fit to experimental data using the AF model. Right: fit to room temperature experimental data using the OW model. Plots consider saturated response after 12 cycles for completely reversed strain amplitudes of $1 \%$ and $1.8 \%$.

Figure 2 compares the experimental uniaxial cyclic stress-strain data of Arcari [15] and simulation results for each of the two slip system constitutive models; corresponding model parameters are listed in Table 1. Both models adequately match the shape of the experimental hysteresis loops used for calibration. These results could only be achieved by introducing kinematic hardening relations at the 
slip system level. Indeed, intergranular interactions alone cannot reproduce the necessary levels of back stress in the hysteresis behavior. This contrasts with modeling the response of duplex Ti-6Al-4V [16-17], for example, in which the low symmetry HCP alpha phase interacts with dual alpha-beta phase colonies to produce suitable macroscopic cyclic stress-strain responses without the need for significant slip system level kinematic hardening.

Table 1. Summary of the constitutive model parameters for Al 7075-T6. Note that $m_{1}=m_{2}$ for the OW model.

\begin{tabular}{|c|c|c|c|c|c|c|c|}
\hline Model & $\boldsymbol{M}$ & $\begin{array}{c}\boldsymbol{G}_{\boldsymbol{o}} \\
(\mathbf{M P a})\end{array}$ & $\begin{array}{c}\boldsymbol{h}_{1} \\
(\mathbf{M P a})\end{array}$ & $\boldsymbol{r}_{\mathbf{1}}$ & $\begin{array}{c}\boldsymbol{h}_{\mathbf{2}} \\
(\mathbf{M P a})\end{array}$ & $\boldsymbol{r}_{\mathbf{2}}$ & $\begin{array}{c}m_{1} \\
m_{2}\end{array}$ \\
\hline AF & 150 & 130 & $7.56 \times 10^{4}$ & 720 & - & - & - \\
\hline OW & 75 & 35 & $2 \times 10^{6}$ & $2 \times 10^{4}$ & $1.35 \times 10^{5}$ & 1421 & 200 \\
\hline
\end{tabular}

Figure 3 illustrates the peak stress vs. peak strain response over a span of applied strain amplitudes considered for calibration. The experimental data in Figure 3 were obtained by Renard et al. [18] and correspond to completely reversed, constant amplitude, strain-controlled uniaxial loading for individual experiments on polycrystalline specimens for applied strain amplitudes over a range from $0.2 \%$ to $2 \%$.

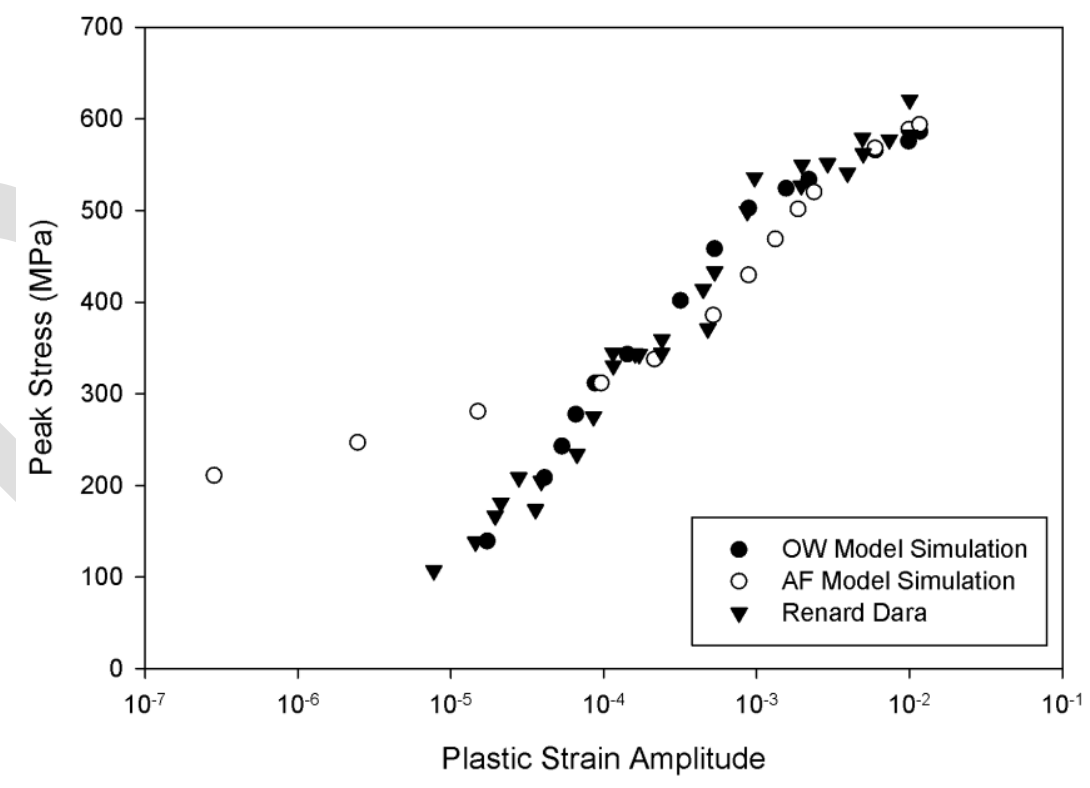

Figure 3. Experimental data for polycrystal peak stress versus plastic strain amplitude [18] compared to results of simulations using the $O W$ and AF models.

The semi-log plot in Figure 3 reveals close agreement between the plastic strain amplitudes predicted by the OW model and the experimental data over the entire range of peak stresses considered. For the AF model, however, the simulated plastic strain amplitude and the experimental 
results diverge significantly below a peak stress value of $280 \mathrm{MPa}$ (corresponding to an applied strain amplitude of $0.4 \%$ ). The OW model captures plastic strain amplitudes at and below applied strain amplitudes of $0.4 \%$ through the use of a much lower value of drag stress ( $35 \mathrm{MPa}$ compared to $130 \mathrm{MPa}$ in the AF model) coupled with a very rapidly evolving first back stress term. The use of a multiple component back stress evolution in the OW model (Eqn. (10)) decouples the back stress response into short range components (quickly evolving terms that control the response at small cyclic strains) and long range components (slowly evolving terms that control the response at larger cyclic strains). This attribute provides a key capability to model both overall hysteresis loop shape and the ratcheting rate, as discussed in the next section. The ability to reproduce cyclic plastic strains at low strain amplitudes is critical for modeling fatigue crack formation and early growth in the high cycle fatigue regime. Furthermore, varying the value of $m_{i}$ for both back stress components within the OW model has only a small effect on the simulated cyclic stress-strain response under completely reversed loading. It is observed that simulations using values for $m_{1}=m_{2}>30$ produce nearly indistinguishable results.

\section{Model Response to Asymmetric Cyclic Loading}

Considering Figures 2 and 3, the OW model is able to reproduce the completely-reversed cyclic stress-strain response at low strain amplitudes more accurately than the AF model. A more demanding assessment of the models should also consider the responses related to progressive cyclic plastic strain accumulation (ratcheting) for stress-controlled cyclic loading under non-zero mean stress, as well as the response of mean stress relaxation under strain-controlled loading with mean strain. Surprisingly, almost no experimental data are available in the open literature for asymmetric strain- or loadcontrolled cycling in the form of complete hysteresis loops (like those used to fit the completely reversed cyclic response) for Al 7075-T6. Experimental mean stress relaxation data obtained by Arcari et al. [19] were used to assess the accuracy of the simulated cyclic stress-strain response in the presence of a mean stress or strain, and to fit the value of $m_{i}$ used in the OW model, as that parameter controls these higher order responses.

A series of simulations were conducted with the OW model with $m_{i}$ values ranging between 10 and 400 under uniaxial strain-controlled cycling with an imposed strain amplitude of $0.4 \%$ and a mean strain, $\varepsilon_{\text {mean }}=1.4 \%\left(\mathrm{R}_{\varepsilon}=\varepsilon_{\min } / \varepsilon_{\max }=0.556\right)$, for a total of 100 computational cycles. The simulations were compared with results of the AF model for the same mesh and loading conditions. The applied strain ratio of $\mathrm{R}_{\varepsilon}=0.556$ produced an initial equivalent stress ratio, $\mathrm{R}_{\sigma}{ }_{\sigma} \approx 0$; as cycling progressed, the equivalent stress ratio decreased slightly by virtue of mean stress relaxation. Cyclic plastic strain ratcheting also occurred. Figure 4 presents the stress versus plastic strain responses for the first three computational cycles for both the AF model and the OW model with $m_{1}=m_{2}=400$, the largest value of $m_{i}$ considered. 


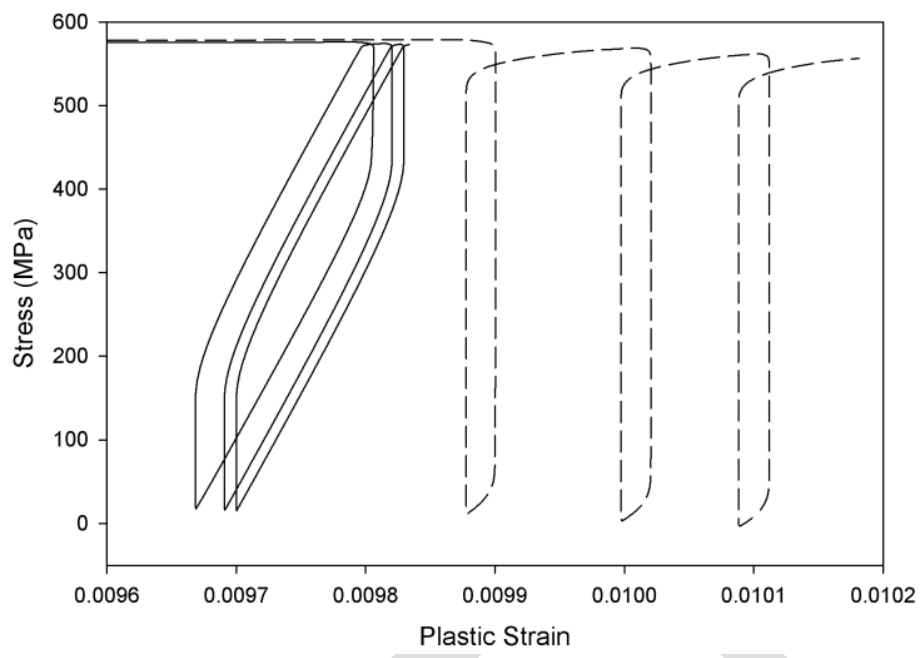

Figure 4. Comparison of unloading/reloading stress versus plastic strain response for three complete computational cycles for $\varepsilon_{\mathrm{a}}=\mathbf{0 . 4 \%}, \mathrm{R}_{\varepsilon}=\mathbf{0 . 5 5 6}$ between the AF model (dashed line) and the OW model with $m_{1}=m_{2}=400$ (solid line).

Several key differences between the responses of the models are apparent in Figure 4. Perhaps most striking is the difference in degree of ratcheting predicted by the models. The AF model predicts ratchet strain per cycle approximately five times larger than the cyclic plastic strain range, while the OW model predicts ratcheting strains that are only a small fraction of the predicted cyclic plastic strain range, thus producing the overlapping hysteresis loops. In addition, the cyclic yield levels suggest that the back stress is significantly underestimated by the AF model. Note that the OW model predicts a larger cyclic plastic strain range under these loading conditions, in agreement with the experimental results shown in Figure 3.

Differences in the rate and extent of mean stress relaxation between the two models are difficult to observe over the three cycles shown in Figure 4. Therefore, Figure 5 plots mean stress relaxation data for Al 7075-T6 measured by Arcari et al. [19] against simulated results using the two models over 100 computational cycles. The data published by Arcari et al. [19] considered 4000 experimental loading cycles, which is on the order of the fatigue life of the specimen when loaded at a strain amplitude of $0.4 \%$ with a mean strain $1.4 \%$. Recall that the model assumes that the hysteresis loops in Figure 2 corresponds to cyclically stable conditions. Thus, the transient response associated with mean stress relaxation for strain-controlled loading with mean strain (or the complementary behavior of cyclic strain ratchetting under stress-controlled loading with mean stress) is governed by the lack of hysteresis loop closure under asymmetric loading conditions. Such a behavior is controlled by the form of the slip system kinematic hardening relation. Accordingly, the experimentally measured mean stress relaxation response has longer range transients in terms of cycle count than those of the computational model, and the initial values of slip system strength in the model at these strain amplitudes are a slightly higher than their initial values of material used in experiments; the model 
should be regarded as an accelerated approach to cyclic stabilization, with more interest devoted to the extent of mean stress relaxation than the observed rate. Accordingly, experimental results in Figure 5 are scaled for comparison such that each computational cycle represents 40 experimental loading cycles. Clearly, access to more complete data regarding initial values and transients for mean stress relaxation of this alloy system would be useful.

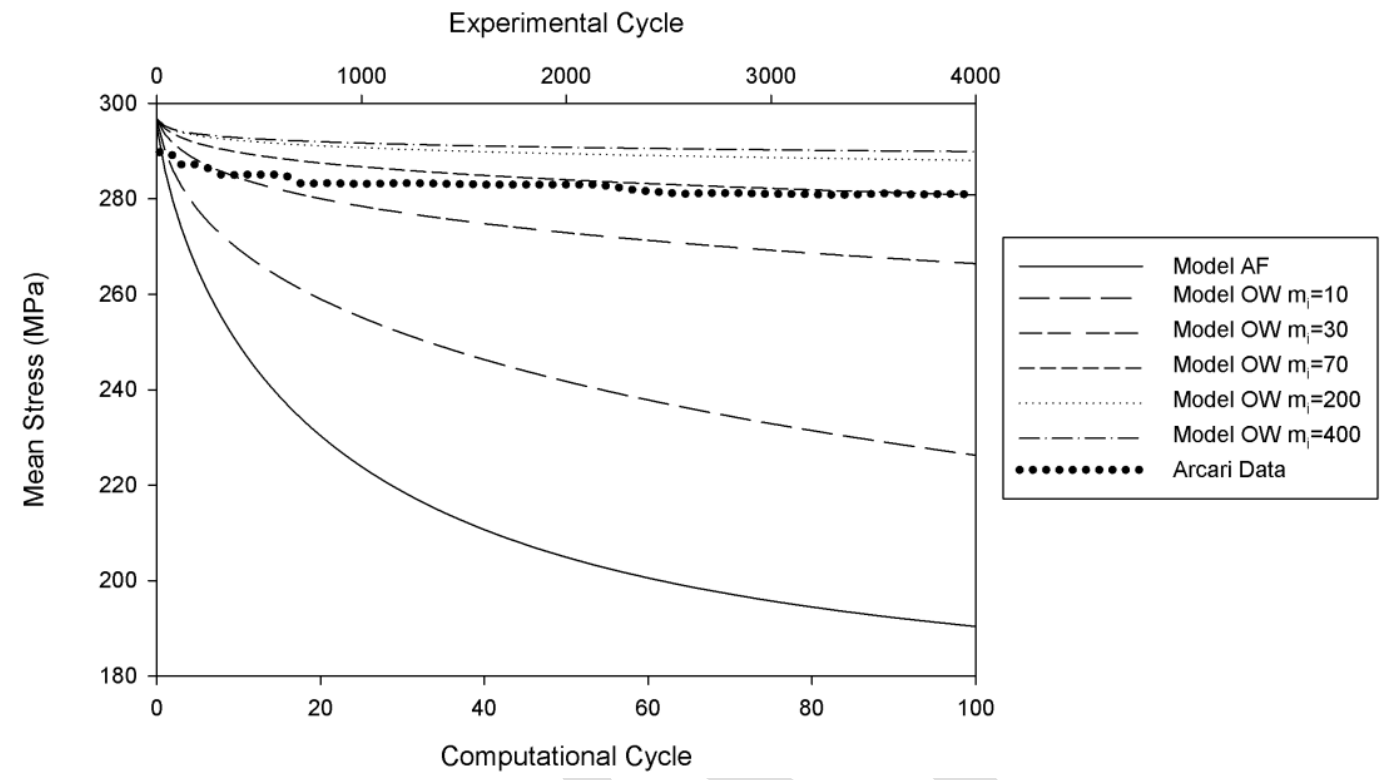

Figure 5. Mean stress relaxation over 100 computational cycles at $\varepsilon_{a}=0.4 \%, \varepsilon_{\text {mean }}=1.4 \%$, and $R_{\varepsilon}=$ 0.556 strain-controlled uniaxial cycling.

Both the AF and OW models result in an initial mean stress that is higher than the experimental results reported by Arcari et al. [19], as shown in Figure 5, but predict a wide range of mean stress relaxation behavior depending on model parameters in the OW model. Figure 5 shows that an increase in $m_{i}$ reduces the extent of mean stress relaxation, but has little effect on the initial mean stress produced by initial straining. Note that the AF model dramatically over predicts the degree of mean stress relaxation by almost $100 \mathrm{MPa}$ (even though the overall hysteresis loop shape was captured under completely reversed loading), while the OW model with $m_{1}=m_{2}=70$ matches the experimentally observed mean stress at the end of the simulation. Comparison of the mean stress relaxation over 100 computational cycles predicted by the OW model for different $m_{i}$ values with experimental measurements indicates that $m_{1}=m_{2}=200$ provides the best agreement with the experimentally measured degree of mean stress relaxation, but values of $m_{1}=m_{2}$ ranging from 100 to 400 all result in simulated mean stress relaxation that is of the same order of magnitude as the experimental data. The AF model, by contrast, is clearly in error by an order of magnitude in its over-prediction of the extent of mean stress relaxation.

Figure 6 presents a semi-log plot of the decay of ratcheting strain per cycle over 100 computational cycles for the AF and OW models with varying values of $m_{i}$ for the OW model, for the 
loading conditions $\varepsilon_{\mathrm{a}}=0.4 \%, \varepsilon_{\text {mean }}=1.4 \%$, and $\mathrm{R}_{\varepsilon}=0.556$. Figure 6 illustrates a decreasing ratcheting strain with increasing $m_{i}$, as well as the large reduction in ratcheting rate predicted by the OW model compared to the AF model. Clearly, models intended for use in computing surrogate driving force measures for intergranular fatigue crack formation at the individual grain level that include ratchet strain accumulation [20] will be sensitive to the slip system back stress constitutive law for Al 7075-T6. Moreover, Figure 3 demonstrates that it is also necessary to consider more sophisticated slip system kinematic hardening models such as the OW model to compute local driving force measures based on cyclic plastic strain range for transgranular fatigue crack formation. Another interesting feature in Figure 6 is the crossover around cycle 60 in predicted ratcheting strain per cycle by the AF model and OW model with $m_{1}=m_{2}=10$. This indicates the tendency of the OW model to approach a saturated ratcheting strain per cycle in fewer cycles than the AF model.

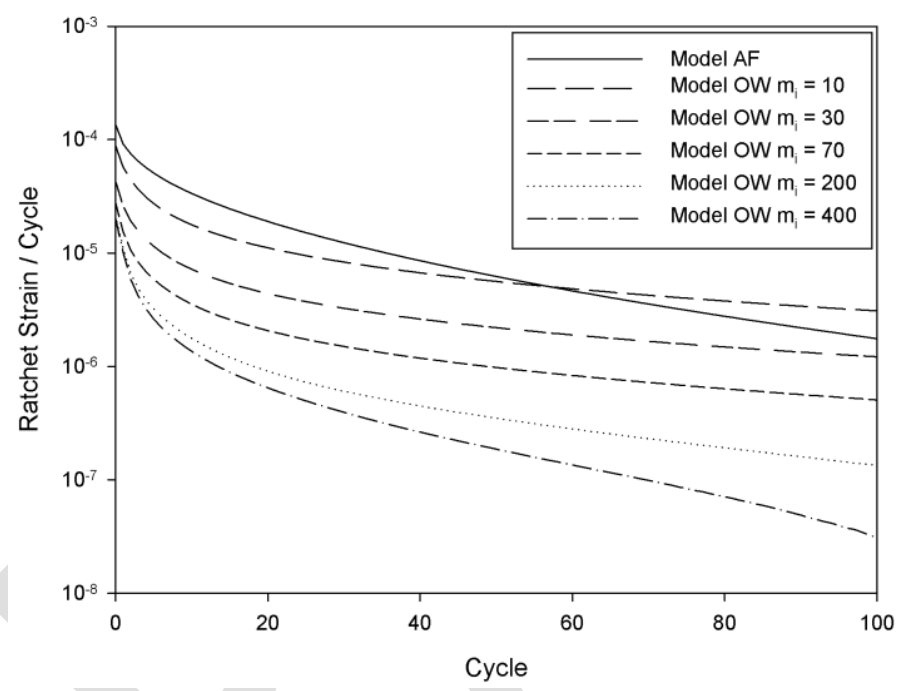

Figure 6. Ratcheting strain per cycle over 100 computational cycles for $\varepsilon_{a}=0.4 \%, \varepsilon_{\text {mean }}=1.4 \%$, and $R_{\varepsilon}=$ 0.556 strain-controlled cyclic loading.

\section{Model Performance at Various Applied Strain Amplitudes and Mean Strains}

Additional simulations were conducted to evaluate the performance of the OW model over a range of applied cyclic strain amplitudes and mean strains selected to match those considered by Arcari et al. [19]. All of these simulations were conducted with uniaxial strain-controlled loading conditions and for 100 computational cycles using the same representative microstructure instantiation (Figure 1) and $m_{1}=m_{2}=70$. The mean stress relaxation results from these simulations are compared to data from Arcari et al. [17] in Figure 7, with simulation data represented by dashed lines, and the experimental data by solid lines. The experimental cycle data are scaled by the reported fatigue life of the specimen, and for the simulated mean stress relaxation data 100 computational cycles is taken to correspond to the specimen half-life, allowing all data to be plotted together. 


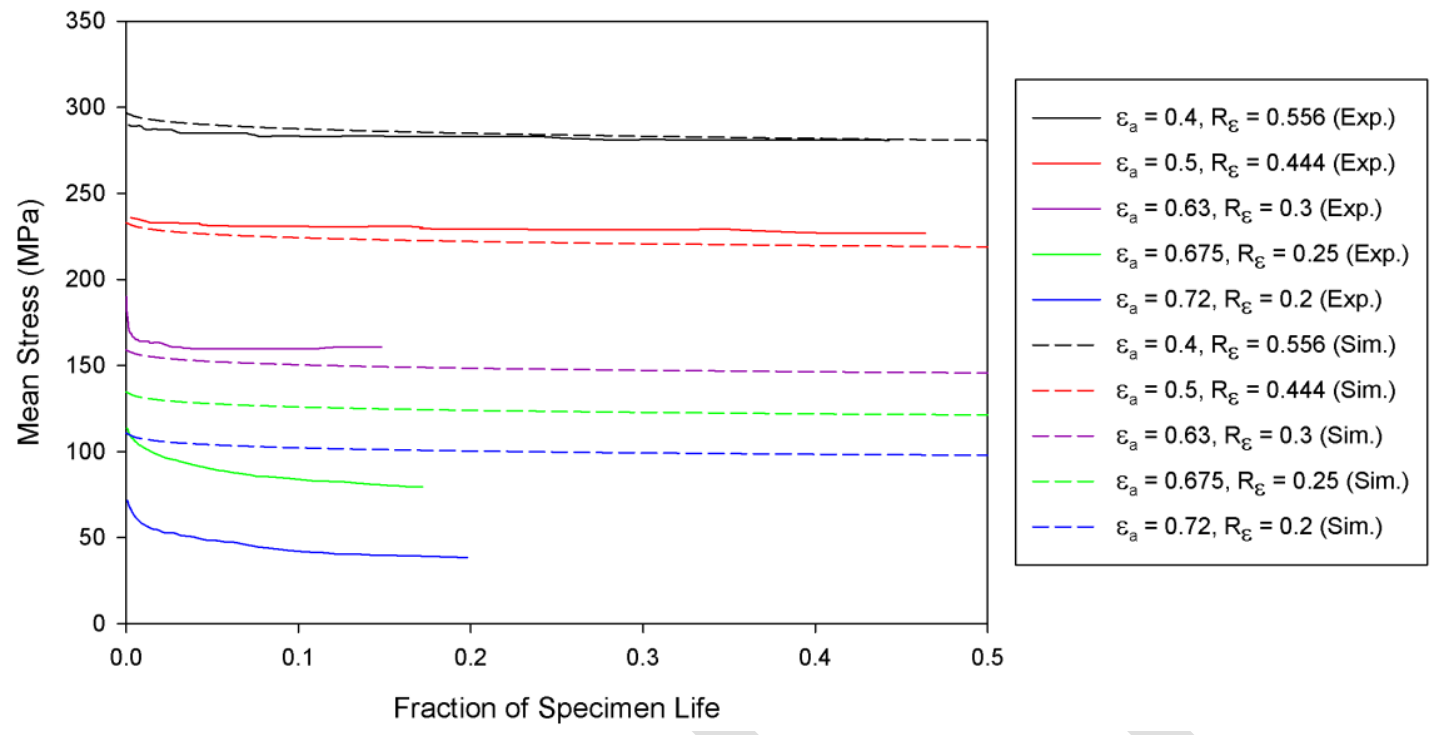

Figure 7. Comparison of simulations (dashed lines) using model $O W$ with $m_{i}=70$ to experimental data of Arcari et al. [19] (solid lines).

In the regime of applied strain amplitudes between $0.63 \%$ and $0.4 \%$, the agreement between the experimental results of Arcari et al. [19] and the simulations is adequate, with absolute errors of less than $10 \mathrm{MPa}$. However, at applied strain amplitudes of $0.675 \%$ and $0.72 \%$, agreement between the model and experimental results diminishes, with absolute errors in excess of $50 \mathrm{MPa}$. This is obviously due to the lack of consideration of transient cyclic softening behavior at this higher applied strain amplitudes. Of course, fatigue life is very short at these higher amplitudes.

\section{Discussion}

The two slip system level kinematic hardening relations discussed in this paper were examined as part of an effort to model microstructurally small crack formation and early growth in Al 7075-T6. Such an application demands accurate prediction of local plastic strains within individual grains under HCF loading conditions, in which the macroscopic total strain range is comparable in magnitude to the macroscopic elastic strain range. We argue that consideration of cyclic strain ratcheting and mean stress relaxation under asymmetric applied loading at low strain amplitudes is an effective surrogate response for local, grain level behavior, compared to overall stress-strain behavior. Of course, in situ experimental measurement of grain level cyclic deformation responses via digital image correlation, for example, would provide even more information for comparison, but measurement errors are challenging in this low amplitude regime of loading and only the sample surface can be accessed. In this study, the OW model is superior to the AF model, due to its capability to better capture overall hysteresis response, cyclic plastic strain range, and mean stress relaxation response, at small applied strain amplitudes typical of the HCF regime. Comparison to experimental data showed that the OW model was capable of accurately modeling ratcheting and mean stress relaxation behavior observed experimentally for this alloy at the applied strain amplitudes of interest. 
The improved agreement with experimental data at small applied strain amplitudes offered by the OW model stems primarily from the multiple component back stress implementation which partitions the slip system level back stress evolution into short and long term components. Multiple component back stress implementations of the AF model are common in the literature for $\mathrm{J}_{2}$ plasticity at the level of the overall polycrystalline response, going back to early works of Chaboche [5-7], Ohno and Wang [2, 21-22], McDowell [4, 23], and Hassan and Kyriakides [24]. Thus, the AF and OW models differ in terms of the nonlinear dependence on the magnitude of the back stress in the dynamic recovery term $\left(2^{\text {nd }}\right.$ term on RHS in Eqn. (10)). This can be seen through manipulation of a single back stress version of Eqn. (10) of the form

$$
\dot{\chi}^{\alpha}=h \dot{\gamma}^{\alpha}-c\left|\chi^{\alpha}\right|^{m+1}\left|\dot{\gamma}^{\alpha}\right| \operatorname{sgn}\left(\chi^{\alpha}\right)
$$

where $c=\frac{r}{b^{m}}$. We can further generalize this approach by assuming that the dynamic recovery term is a general function $F\left(\chi^{\alpha}, \gamma^{\alpha}, \ldots\right)$, i.e.,

$$
\dot{\chi}^{\alpha}=h \dot{\gamma}^{\alpha}-F\left(\chi^{\alpha}, \gamma^{\alpha}, \ldots\right)\left|\dot{\gamma}^{\alpha}\right|
$$

Along these lines, it is clear that the AF and OW models are based on the same generic back stress formulation with different choices for the function $F\left(\chi^{\alpha}, \gamma^{\alpha}, \ldots\right)$. Other researchers have considered crystal plasticity approaches with various nonlinear dynamic recovery terms (cf. [25-27]). The origin and phenomenology of $F\left(\chi^{\alpha}, \gamma^{\alpha}, \ldots\right)$, and indeed of the nonlinear dynamic term itself, can be traced to the evolution of mesoscale dislocation substructures such as cells. For example, following Berveiller and Zaoui [28] and Sauzay [29], the back stress under single slip depends on dislocation wall fraction $\left(f_{w}\right)$, i.e.,

$$
\chi=\frac{f_{w}}{1-f_{w}}(1-\beta) \mu F_{a c c} \gamma
$$

Here, $\beta$ depends on the Eshelby tensor, $\mu$ is the shear modulus, and $\gamma$ is the positive monotonic magnitude of applied shear deformation per cycle. The accommodation factor, $F_{a c c}$, represents the contribution of dislocation structures and may be represented by

$$
F_{a c c} \approx \frac{1}{1+\frac{1}{2} \mu \frac{\gamma}{\tau_{0}}}
$$

The product $\mu \gamma$ is normalized by shear strength factor $\tau_{o}$. Differentiating Eqn. (13) with respect to time yields 


$$
\dot{\chi}=\frac{f_{w}}{1-f_{w}}(1-\beta) \mu F_{a c c} \dot{\gamma}+\frac{f_{w}}{1-f_{w}}(1-\beta) \mu \gamma \dot{F}_{a c c}
$$

where

$$
\dot{F}_{a c c} \approx \frac{1}{\left(1+\frac{1}{2} \mu \frac{\gamma}{\tau_{0}}\right)^{2}} \frac{1}{2} \mu \frac{\dot{\gamma}}{\tau_{0}}
$$

This leads to

$$
\dot{\chi}=\frac{f_{w}}{1-f_{w}}(1-\beta) \mu F_{a c c} \dot{\gamma}-\frac{f_{w}}{1-f_{w}}(1-\beta) \mu F_{a c c} \gamma \frac{1}{\left(1+\frac{1}{2} \mu \frac{\gamma}{\tau_{0}}\right)} \frac{1}{2} \mu \frac{\dot{\gamma}}{\tau_{0}}
$$

Equivalently, we may write

$$
\dot{\chi}=\frac{f_{w}}{1-f_{w}}(1-\beta) \mu F_{a c c} \dot{\gamma}-\frac{1}{2} \mu \frac{F_{a c c}}{\tau_{0}} \chi \dot{\gamma}
$$

Assuming $\gamma$ is positive and monotonically increasing, this can be expressed as

$$
\dot{\chi}=A_{b} \dot{\gamma}-A_{c} \chi|\dot{\gamma}| \text { with } A_{b}=\frac{f w}{1-f w}(1-\beta) \mu F_{a c c} \text { and } A_{c}=\frac{1}{2} \mu \frac{F_{a c c}}{\tau_{0}}
$$

Equation (19) is a hardening-dynamic recovery form of back stress evolution in which $A_{b}$ and $A_{c}$ depend on the accommodation factor, which in turn depends on $\chi$ and $\gamma$. The additional simplification of considering $F_{a c c}$ as constant yields the Frederick-Armstrong formulation. In cases with significant ratcheting caused by the evolution of the dislocation walls, $F_{a c c}$ may change significantly; even over a given loading cycle, dislocation rearrangement within the walls can give rise to complex unloadingreloading phenomena. In such cases, nonlinear dynamic recovery terms, such as in the OW model, provide additional flexibility to represent the evolution of $F_{a c c}$, which reflects dynamic recovery processes associated with mesoscale dislocation structures.

As discussed earlier, it is often tacitly assumed that the intergranular interactions are somehow responsible for modifying the polycrystalline response to account for proper description of ratcheting. However, this does not appear to be the case for Al 7075-T6, which is characterized by slip in a high symmetry FCC crystal structure, relatively low elastic anisotropy, and propensity for dislocation cross slip. This understanding has important consequences for microstructure-sensitive fatigue modeling [3032] that purports to assess local driving forces within grains or near interfaces to form and grow small fatigue cracks. The importance of slip system kinematic hardening rules in this regard has not always been acknowledged in such studies (cf. [20]). Moreover, Feaugas and colleagues [33-34] have clearly demonstrated the importance of slip system back stress on cyclic ratcheting by virtue of internal 
stresses associated with intragranular dislocation substructure. These studies are in agreement with our interpretation of Eqn. (19), which suggests that OW model is capable of representing the dynamic recovery of mesoscale structures.

One complication introduced by the Ohno-Wang type kinematic hardening formulation is that the evolution of the back stress with respect to changes in the sign of $\dot{\gamma}^{\alpha}$ occurs rapidly whenever any of the back stress terms approach saturation, especially for values of $m_{i}>70$. This rapid evolution can present challenges to convergence, requiring additional strain or time step sub-incrementation, and thus longer simulation times when compared to simulations conducted using the AF model. Computational times for the OW model relative to the AF model increase from about $10 \%$ for $m_{i}$ values less than 70 to about $25 \%$ for higher values of $m_{i}$. Considering the significant improvements of the OW model in modeling cyclic plastic response at low stress or strain amplitudes, as well as ratcheting and mean stress relaxation, this additional computational cost appears to offer an acceptable tradeoff.

\section{Conclusions}

Motivated by the desire to model cyclic plastic strain response at the grain level in polycrystals to support microstructure-sensitive computational exploration of the formation and early growth of microstructurally small fatigue cracks [30-32], we have examined two crystal plasticity constitutive models for slip system nonlinear kinematic hardening of the cyclically stable stress-strain response of Al 7075-T6. Kinematic hardening was introduced at the slip system level as a result of early exploration that indicated its necessity in matching hysteresis loop shapes and mean stress relaxation behavior; intergranular interactions were otherwise too weak for this high slip symmetry, low elastic anisotropy FCC alloy system. The performance of the models in simulating cyclic stress-strain behavior was compared under symmetric and asymmetric strain-controlled cyclic loading for multiple applied strain ranges. Although each of the models was calibrated to the same completely reversed, uniaxial cyclic stress-strain data, there are substantial and important differences in the responses of the models at small applied strain amplitudes and when loaded cyclically with a mean strain. Under strain-controlled loading with mean strain, the AF model both dramatically over-predicted the degree of mean stress relaxation and exhibited significant ratcheting strains that are known to be an artifact of the model form. Much better agreement was obtained via implementation of a multiple back stress Ohno-Wang type hardening law with nonlinear dynamic recovery at the slip system level. To summarize, the advantages of a two-term Ohno-Wang type formulation of the back stress evolution law over the classical Armstrong-Frederick model for Al 7075-T6 at the slip system level are:

- Enhanced ability to capture the magnitude of the experimentally observed cyclic plastic strain range over a larger range of applied strain amplitudes, most notably for low amplitudes in the HCF regime.

- Increased ease of fitting experimental responses.

- Ability to match experimental mean stress relaxation data under asymmetric strain-controlled cycling with mean strain through selection of $m_{i}$, which has only a minor effect on the shape of the completely reversed hysteresis loops. 
- Ability to rapidly approach a nearly saturated value of cyclic plastic strain range when loaded with an imposed mean strain.

Despite slight increases in computational cost, the adoption of an Ohno-Wang type of back stress evolution law significantly enhances the predictive accuracy of the model under both completely reversed and asymmetric loading conditions. To our knowledge, this is the first time such a general nonlinear dynamic recovery back stress formulation has been employed in a crystal plasticity model at the slip system level. More general forms of slip system level hardening-nonlinear dynamic recovery relations for back stress evolution were discussed, motivated by slip system interactions with meososcale dislocation substructures.

\section{Acknowledgements}

The authors are grateful for the support provided by Integrated Systems Solutions, Inc. (Technical Monitor: Dr. Nam Phan, NAVAIR). 


\section{References}

1. Abaqus unified FEA (2016) Dassault Systèmes Americas Corp., Waltham, MA.

2. Frederick, C.O. and P.J. Armstrong (1966) A mathematical representation of the multiaxial Bauschinger effect. G.E.G.B. Report RD/B/N 731.

3. Ohno, N. and J.D. Wang (1993) Kinematic hardening rules with critical state of dynamic recovery, Part I: Formulation and basic features for ratchetting behavior. International Journal of Plasticity 9:375-390.

4. McDowell, D.L. (1995) Stress state dependence of cyclic ratchetting behavior of two rail steels. International Journal of Plasticity 11(4):397-421.

5. Chaboche, J.L. and D. Nouailhas (1989) Constitutive Modeling of Ratchetting Effects-Part I: Experimental Facts and Properties of the Classical Models. Journal of Engineering Materials and Technology 111:384-392.

6. Chaboche, J.L. and D. Nouailhas (1989) Constitutive Modeling of Ratchetting Effects-Part II: Possibilities of Some Additional Kinematic Rules. Journal of Engineering Materials and Technology 111:409-416.

7. Chaboche, J.L. (1991) On some modifications of kinematic hardening to improve the description of ratchetting effects. International Journal of Plasticity 7:661-678.

8. Turkmen, H.S., R.E. Loge, P.R. Dawson, and M.P. Miller (2003) On the mechanical behaviour of AA 7075-T6 during cyclic loading. International Journal of Fatigue 25(4):267-281.

9. Bozek, J.E., J.D. Hochhalter, M.G. Veilleux, M. Liu, G. Heber, S.D. Sintay, A.D. Rollett, D.J. Littlewood, A.M. Maniatty, H. Weiland, R.J. Christ, J. Payne, G. Welsh, D.G. Harlow, P.A. Wawrzynek, and A.R. Ingraffea (2008) A geometric approach to modeling microstructurally small fatigue crack formation: I. Probabilistic simulation of constituent particle cracking in AA 7075T651. Modelling and Simulation in Materials Science and Engineering 16(6):065007.

10. Hochhalter, J.D., Littlewood, D.J., Christ, R.J., Jr., Veilleux, M.G., Bozek, J.E., Ingraffeas, A.R., and Maniatty, A.M., (2010) A geometric approach to modeling microstructurally small fatigue crack formation: II. Physically based modeling of microstructure-dependent slip localization and actuation of the crack nucleation mechanism in AA 7075-T651. Modelling and Simulation in Materials Science and Engineering 18(4):045004.

11. Littlewood, D. and A. Maniatty (2005) Multiscale modeling of crystal plasticity in Al 7075-T651. Proc. VIII International Conference on Computational Plasticity Fundamentals and Applications (COMPLAS VIII), Barcelona, Spain, 618-621.

12. Wang, L., S. Daniewicz, M. Horstemeyer, S. Sintay, and A. Rollett (2009) Three-dimensional finite element analysis using crystal plasticity for a parameter study of fatigue crack incubation in a 7075 aluminum alloy. International Journal of Fatigue 31(4):659-667.

13. Johnston, S.R., G.P. Potirniche, S.R. Daniewicz, and M.F. Horstemeyer (2006) Three-dimensional finite element simulations of microstructurally small fatigue crack growth in 7075 aluminium alloy. Fatigue \& Fracture of Engineering Materials and Structures 29(8):597-605.

14. Asaro, R.J. (1983) Crystal plasticity. ASME J. Appl. Mech. 50:921-934.

15. Arcari, A. (2010) Enhanced strain-based fatigue methodology for high strength aluminum alloys. PhD thesis, Virginia Polytechnic Institute and State University, Blacksburg, VA.

16. Bridier, F., D.L. McDowell, P. Villechaise, and J. Mendez (2009) Crystal plasticity modeling of slip activity in Ti-6Al-4V under high cycle fatigue loading. International Journal of Plasticity 25(6):1066-1082.

17. Zhang, M., J. Zhang, and D.L. McDowell (2007) Microstructure-based crystal plasticity modeling of cyclic deformation of Ti-6Al-4V. International Journal of Plasticity 23(8):1328-1348. 
18. Renard, A., A.S. Cheng, R. De La Veaux, and C. Laird (1983) The Cyclic Stress-Strain Response of a Polycrystalline Al-Zn-Mg Alloy and Commercial Alloys Based on this System. Materials Science and Engineering 60:113-120.

19. Arcari, A., R. De Vita, and N.E. Dowling (2009) Mean stress relaxation during cyclic straining of high strength aluminum alloys. International Journal of Fatigue 31(11-12):1742-1750.

20. Hochhalter, J.D. (2010) Finite element simulations of fatigue crack stages in AA 7075-T651 microstructure, PhD Thesis, Cornell University, Ithaca, NY.

21. Ohno, N. and J.D. Wang (1991) Nonlinear kinematic hardening rule: proposition and application to ratchetting problems. Trans. SMiRT 11, Vol. L, Tokyo, Japan, 481-46.

22. Ohno, N. and J.D. Wang (1992) Basic features of kinematic hardening models with critical state of dynamic recovery in relation to multiaxial ratchetting behavior. Proc. MECAMAT '92, Int. Seminar on Multiaxial Plasticity, Cachan, France.

23. McDowell, D.L. (1992) Description of nonproportional cyclic ratchetting behavior. Proc. MECAMAT '92, Int. Seminar on Multiaxial Plasticity, Cachan, France.

24. Hassan, T. and S. Kyriakides (1992) Ratcheting in cyclic plasticity, part I: uniaxial behavior. International Journal of Plasticity 8(1):91-116.

25. Castelluccio, G.M. and D.L. McDowell (2013) Effect of annealing twins on crack initiation under high cycle fatigue conditions. Journal of Materials Science 48(6):2376-2387.

26. Kang, G., O.T. Bruhns, and K. Sai (2011) Cyclic polycrystalline visco-plastic model for ratchetting of 316L stainless steel. Computational Materials Science 50(4):1399-1405.

27. Dong, Y., G. Kang, and C. Yu (2014) A dislocation-based cyclic polycrystalline visco-plastic constitutive model for ratchetting of metals with face-centered cubic crystal structure. Computational Materials Science 91:75-82.

28. Berveiller, M. and A. Zaoui (1978) An extension of the self-consistent scheme to plasticallyflowing polycrystals. Journal of the Mechanics and Physics of Solids 26(5):325-344.

29. Sauzay, M. (2008) Analytical modelling of intragranular backstresses due to deformation induced dislocation microstructures. International Journal of Plasticity 24(5):727-745.

30. McDowell, D.L. (2007) Simulation-based strategies for microstructure-sensitive fatigue modeling. Materials Science and Engineering A 468-470:4-14.

31. McDowell, D.L. and F.P.E. Dunne (2010) Microstructure-sensitive computational modeling of fatigue crack formation. International Journal of Fatigue 32(9):1521-1542.

32. Castelluccio, G.M., W.D. Musinski, and D.L. McDowell (2014) Recent developments in assessing microstructure-sensitive early stage fatigue of polycrystals. Current Opinion in Solid State and Materials Science 18(4):180-187.

33. Feaugas, X. and H. Haddou (2007) Effects of grain size on dislocation organization and internal stresses developed under tensile loading in fcc metals. Philosophical Magazine 87(7):989-1018.

34. Feaugas, X. (1999) On the origin of the tensile flow stress in the stainless steel AISI $316 \mathrm{~L}$ at $300 \mathrm{~K}$ : back stress and effective stress. Acta Materialia 47(13):3617-3632. 
2017-01-22

Sensitivity of polycrystal plasticity to slip

system kinematic hardening laws for Al 7075-T6

Hennessey, Conor

Elsevier

Conor Hennessey, Gustavo M. Castelluccio, David L. McDowell, Sensitivity of polycrystal plasticity to slip system kinematic hardening laws for Al 7075-T6, Materials Science and Engineering: A, Volume 687, 27 February 2017, Pages 241-248

http://dx.doi.org/10.1016/j.msea.2017.01.070

Downloaded from Cranfield Library Services E-Repository 